\title{
Sangrado intraoperatorio de amigdalectomía en población pediátrica. Influencia de factores quirúrgicos y anestésicos
}

\author{
Intraoperative bleeding of tonsillectomy in pediatric \\ patients. Effects of surgical and anesthesia technique
}

Dagoberto Ojeda D. ${ }^{1, a}$, Isabel Carranza L. b , Álvaro Jorquera S. ${ }^{5}$, María Luz Rubio V. 2,a, Mariela Agurto V. ${ }^{3, a}$, Tomás Icaran P. ${ }^{4, a}$, Patricia Cisternas M. ${ }^{a}$

\begin{abstract}
Introduction: Tonsillectomy with or without adenoidectomy is one of the most frequent surgeries in the pediatric population. It has become predominantly an outpatient procedure. Therefore, it is of utmost importance identifying the factors that influence the intraoperative bleeding to prevent postoperative complications and rehospitalization. Material and Methods: An observational cross-sectional study was carried out. Patients between 1 and 14 years old that underwent to tonsillectomy with or without adenoidectomy since November 2015 to May 2017 were included. 709 cases were evaluated. Intraoperative bleeding was assessed by the volumetric method. A multivariate analysis was performed using a generalized linear regression model. Results: The average intraoperative bleeding was estimated in $1.9 \mathrm{ml} / \mathrm{kg}(95 \% \mathrm{Cl}: 1.7$ -2.05). The use of propofol (30\% increase in bleeding) and surgical time $(2 \%$ increase for every minute) were risk factors. The use of electrocautery was associated with a $50 \%$ decrease in bleeding in comparison with conventional dissection $(p=0.001)$. Conclusions: The use of propofol and a prolonged surgical
\end{abstract}

Key words:

Bleeding, propofol, child, tonsillectomy

\footnotetext{
Magíster Bioestadística, Universidad de Chile.

Magíster de Educación en Salud(C), Universidad de Chile.

Magíster en Bioética, Universidad de Chile.

Anestesiólogo, Universidad de los Andes.

Médico Cirujano, Universidad de los Andes.

Servicio de Anestesiología, Clínica Dávila.

Servicio de Otorrinolaringología, Clínica Dávila.
}

Fecha de recepción: 15 de abril de 2020

Fecha de aceptación: 03 de agosto de 2020

\section{ORCID}

https://orcid.org/0000-0001-8160-9720

Conflicto de interés: Ninguno.

Correspondencia:

Dr. Dagoberto Ojeda D.

Servicio de Anestesiología Clínica Dávila

eojedadinamarca@gmail.com 
time were risk factors for intraoperative bleeding. The use of electrosurgery was a protective factor.

\section{RESUMEN}

Introducción: La amigdalectomía con o sin adenoidectomía, es una de las cirugías más frecuente en población pediátrica. Desde hace varios años se ha vuelto una intervención predominantemente ambulatoria, por lo que lograr identificar los factores que influyen en el sangrado intraoperatorio es de suma importancia para prevenir complicaciones postoperatorias y reshospitalización. Material y Método: Se realizó un estudio observacional de corte-transversal. Se incluyó a pacientes entre 1 y 14 años sometidos a amigdalectomía con o sin adenoidectomía entre noviembre de 2015 y mayo de 2017, obteniendo un total de 709 casos evaluados. Se determinó el sangrado intraoperatorio de forma volumétrica. Posteriormente, se realizó un análisis multivariado con un modelo de regresión lineal generalizado. Resultados: Se cuantificó el sangrado intraoperatorio promedio en $1,9 \mathrm{ml} / \mathrm{kg}$ (IC 95\%; 1,7-2,05). El uso de propofol (aumento del $30 \%$ del sangrado) y tiempo quirúrgico (2\% por cada incremento de un minuto) fueron factores de riesgo. Mientras que el uso de electro bisturí se asoció con una disminución del $50 \%$ en relación al no uso $(p=0,001)$. Conclusiones: Fueron factores de riesgo para sangrado intraoperatorio el uso de propofol y un tiempo quirúrgico prolongado. El uso de electrobisturí constituyó un factor protector.

\section{Palabras clave:}

Sangrado,

tonsilectomía,

propofol,

población pediátrica

\section{Introducción}

L a cirugía otorrinolaringológica es el procedimiento más frecuentemente realizado en edad pediátrica, específicamente la amigdalectomía con o sin adenoidectomía, siendo en la mayoría de los casos de carácter ambulatorio[1],[2]. Este procedimiento busca resolver la obstrucción de la vía aérea superior y/o prevenir la recurrencia o cronicidad de los procesos inflamatorios faríngeos[3].

Debido a la compleja anatomía vascular de la amígdala, el sangrado suele ser parte importante de la cirugía, dando cuenta del 5\%-10\% del volumen circulante sanguíneo[4]. A pesar de los numerosos esfuerzos, el sangrado postoperatorio continúa siendo la complicación más común y relevante, presentándose en hasta $10 \%$ de los casos, a menudo dentro de las primeras $24 \mathrm{~h}$ postoperatorias (sangrado inmediato), pudiendo tener resultados catastróficos[5].

El sangrado postoperatorio se relaciona directamente con el sangrado intraoperatorio. En 1996, Myssiorek et al., evaluó los factores de riesgo asociados al sangrado postoperatorio en 1.138 pacientes sometidos a amigdalectomía, y observó que un sangrado intraoperatorio de mayor cuantía se relaciona- ba significativamente con un mayor sangrado en el postoperatorio[6].

La literatura con respecto al sangrado intraoperatorio es limitada. En 1965, Holden et al., estimó dicho sangrado en 128,1 ml en pacientes de 4 a 12 años[7], mientras que estudios más recientes sugieren 92,46 $\mathrm{ml}[8]$ y $73,7-79,8 \mathrm{ml}$ en pacientes de 4 a 9 años[9]. A pesar de ser potencialmente severo, la tasa de transfusión asociada al sangrado intraoperatorio se mantiene baja (0\%-3\%), y a menudo se ve relacionada con alteraciones de la coagulación[10],[11].

Se han descrito múltiples factores asociados al sangrado intraoperatorio, tales como: tipo de patología amigdalina subyacente (presencia de fibrosis, amigdalitis crónica)[9], la técnica quirúrgica (técnica caliente o fría)[12], uso de AINEs como el ketorolaco[13], presencia de coagulopatías previas[14], uso de antifibrinolíticos como el ácido tranexámico[15],[16], procoagulantes como el subgalato[17] y el uso de antibióticos[9]. Estos factores son controvertidos y han sido puestos en duda por algunos autores.

Dado la relevancia del sangrado intraoperatorio en las complicaciones postoperatorias y la inexistencia de datos en nuestra población, se decidió cuantificar la magnitud del sangrado intraoperatorio en amigda- 
lectomía pediátrica y así determinar los factores de riesgo asociados.

\section{Material y Métodos}

Se solicitó la aprobación del comité ético-científico institucional, el cual determinó que no se requería el consentimiento informado de los padres o representantes legales previo al estudio. Se realizó un estudio observacional de tipo corte-transversal entre noviembre de 2015 y mayo de 2017 en un único centro.

Se incluyeron a todos los pacientes menores de 15 años, programados de forma electiva para amigdalectomía con o sin adenoidectomía dentro del período descrito, con el fin de manejar la obstrucción de vía aérea superior y los procesos de amigdalitis recuerrente o crónica. La técnica quirúrgica y anestésica fue elegida por cada equipo quirúrgico, sin cambios en el actuar habitual. No hubo criterios de exclusión.

Previo a la cirugía se recolectó una historia clínica detallada. Se registraron características demográficas (edad, género, peso, ASA), antecedentes personales o familiares de coagulopatía y las pruebas de coagulación preoperatorias habituales (conteo plaquetario, INR y TTPK).

Se utilizó anestesia general en la totalidad de los casos. Se registró el tipo de inducción (inhalatoria vs endovenosa), el tipo de intubación (oral vs nasal) y las drogas utilizadas en el intraoperatorio (fentanilo, propofol, bloqueadores neuromusculares, AINEs, corticoides, ácido tranexámico, antibióticos). La mantención anestésica se realizó con gases inhalatorios (sevofluorano o desfluorano).

La técnica quirúrgica fue determinada por cada equipo. Se registró el tiempo operatorio y el tipo de técnica utilizada (fría vs caliente). Se definió como técnica fría aquella realizada mediante la disección con asa de alambre (disector tipo Daniels), hemostasia con packing, aplicación de subgalato de bismuto y uso de sutura reabsorbible cuando fuese necesario. Se consideró como técnica caliente aquella donde la disección y la hemostasia fueron realizadas con electrobisturí. El uso de procoagulantes tópicos como el subgalato fue determinado por el cirujano a cargo en base a su práctica habitual.

El sangrado intraoperatorio fue estimado de forma volumétrica por el anestesiólogo a cargo del paciente. Se midió la sangre recolectada en el recipiente de aspiración al finalizar la cirugía, descontando la cantidad de suero fisiológico instilado y desestimando el contenido de sangre embebido en tórulas y gasas.

Se calculó un tamaño muestral de 550 pacientes para obtener un adecuado nivel de predicción según lo recomendado por Knofczynski GT[18]. Para su estimación se consideró la necesidad de utilizar un modelo de regresión multivariado que comprendiera al menos 5 variables predictoras.

Debido a que la distribución del sangrado intraoperatorio no presentó una distribución normal (con tendencia a la acumulación de los datos hacia valores bajos), se decidió realizar un análisis mediante un modelo de regresión lineal generalizada (GLM), con familia de distribución gamma y transformación logarítmica de los resultados.

Se procedió a realizar un análisis univariado y aquellas variables que presentaron mayor asociación (determinado por $\mathrm{p}<0,2$ ) fueron incorporadas al análisis multivariado. Posteriormente, se consideraron como significativas aquellas variables con $p<0,05$. Se utilizó STATA 13 para el análisis estadístico.

\section{Resultados}

Se obtuvo información de 709 pacientes sometidos a amigdalectomía con o sin adenoidectomía durante el período descrito, cuyas características demográficas y clínicas se describen en la Tabla 1. El sangrado intraoperatorio promedio fue de $41 \mathrm{ml}$, IC 95\% (37 - 45), con un máximo sangrado de $700 \mathrm{ml}$. Se estandarizó la magnitud del sangrado según $\mathrm{ml} / \mathrm{kg}$ de peso, siendo este de 1,9 ml/kg, IC 95\% $(1,7-2,05)$.

Los resultados del análisis univariado se observan en la Tabla 2. Las variables que demostraron un p-valor $<0,2$, fueron el uso de propofol, tiempo quirúrgico prolongado, amigdalectomía con adenoidectomía (vs amigdalectomía aislada), intubación nasal (vs oral) y uso de electrobisturí.

Las variables que de forma independiente demostraron asociación significativa fueron el uso de propofol (Figura 1), técnica quirúrgica (Figura 2) y tiempo quirúrgico prolongado (Figura 3). Al comparar técnica caliente vs fría, se apreció una disminución de 50\% en el sangrado ( $p=0,001)$, mientras que el uso de propofol aumentó el sangrado en $30 \%$ en comparación al no uso de este fármaco $(p=0,004)$. El sangrado aumentó en $2 \%$ por cada minuto de tiempo quirúrgico (Tabla 3).

\section{Discusión}

Se determinó un sangrado intraoperatorio promedio de $41 \mathrm{ml}$, algo menor a lo observado en otros estudios[7]-[9]. Hubo una gran dispersión a causa de 


\begin{tabular}{|c|c|}
\hline Variable & $(n=709)$ \\
\hline Edad - años *(RI) & $4(3)$ \\
\hline Hombres - \% & 54 \\
\hline Mujeres - \% & 46 \\
\hline Peso - kg* (RI) & $20(9)$ \\
\hline \multicolumn{2}{|l|}{ Clasificación ASA - \% } \\
\hline ASA I & 78 \\
\hline ASA \| & 22 \\
\hline \multicolumn{2}{|l|}{ Pruebas de coagulación } \\
\hline$-I^{*} R^{*}(\mathrm{RI})$ & $1,08(0,12)$ \\
\hline \multicolumn{2}{|l|}{ - TTPK* - seg (RI) 30,1 $(4,4)$} \\
\hline - Recuento plaquetario - 103/uL* (RI) & $330(94)$ \\
\hline \multicolumn{2}{|l|}{ Técnica quirúrgica - \% } \\
\hline - Fría & 94 \\
\hline - Caliente & 6 \\
\hline \multicolumn{2}{|l|}{ Técnica anestésica - \% } \\
\hline - Inducción inhalatoria & 98 \\
\hline - Inducción endovenosa & 2 \\
\hline \multicolumn{2}{|l|}{ Drogas utilizadas - \% } \\
\hline - Sevofluorano & 98,5 \\
\hline - Fentanilo & 100 \\
\hline Dosis - ug/kg* (RI) & $3,5(1,3)$ \\
\hline - Propofol & 39 \\
\hline - Bloqueadores neuromusculares & 6 \\
\hline - Ketoprofeno & 99 \\
\hline - Ketorolaco & 1 \\
\hline - Dexametasona & 100 \\
\hline Dosis - mg/kg* (RI) & $0,1(0,02)$ \\
\hline - Ácido tranexámico & 8 \\
\hline - Otros procoagulantes & 0,7 \\
\hline - Antibióticos & 6,5 \\
\hline \multicolumn{2}{|l|}{ Tipo de intubación - \% } \\
\hline - Intubación nasal & 43,4 \\
\hline - Intubación oral & 56,6 \\
\hline Duración cirugía* - min & $27(10)$ \\
\hline
\end{tabular}

*mediana. Rl: rango intercuartílico.

las diferentes complexiones físicas de los pacientes (8,5-74 kg), por lo que se optó por estandarizar el sangrado por $\mathrm{ml} / \mathrm{kg}$ peso, obteniendo un sangrado intraoperatorio de 1,9 ml/kg. Esta aproximación es de

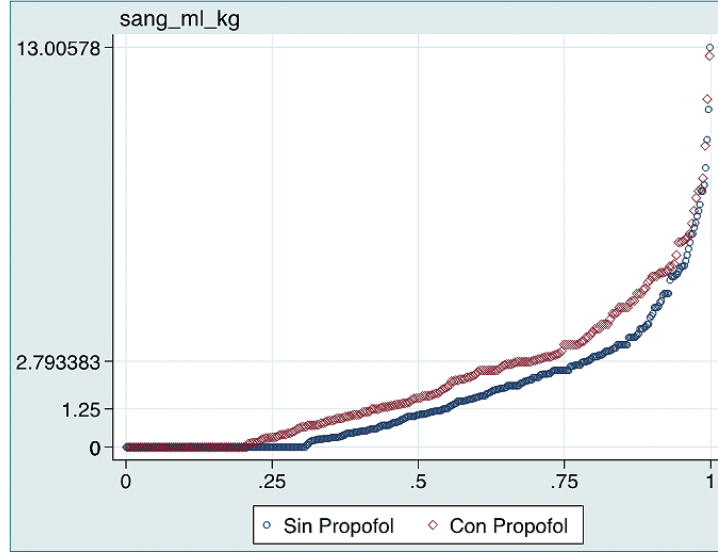

Figura 1. Gráfico Q-Q (cuantil-cuantil). Sangrado intraoperatorio en relación al uso de propofol (rojo) y sin uso de propofol (azul).

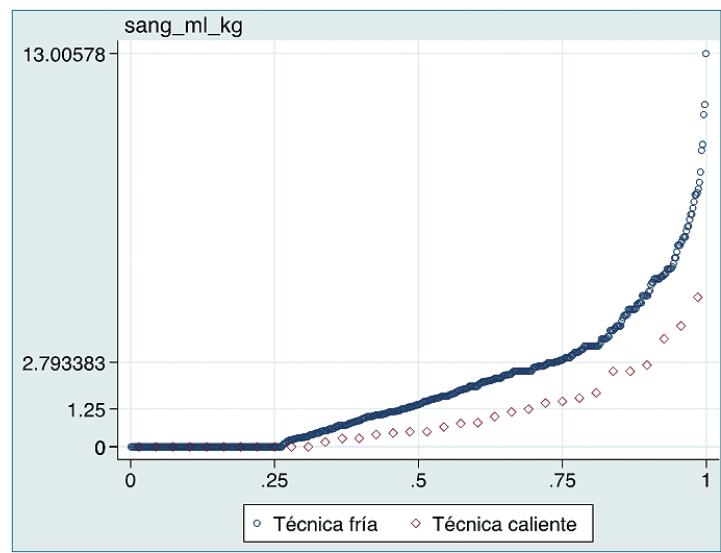

Figura 2. Gráfico Q-Q (cuantil-cuantil) sangrado intraoperatorio en relación a técnica quirúrgica. Se observa un mayor sangrado con técnica fría (azul) en relación con técnica caliente (rojo).

mayor utilidad dado a que la población sometida a esta cirugía fluctúa entre un rango ettario extenso y varía según el centro.

Al estimar el sangrado, existen ciertos factores confundentes que pudieron haber alterado la medición. Se determinó el sangrado de forma volumétrica observando la cantidad de sangre en el receptáculo de aspiración, omitiendo el pesaje de la sangre en gasas y tórulas (método gravimétrico), dado la necesidad de un método expedito y reproducible en el tiempo. Esta determinación pudo haber subestimado el sangrado intraoperatorio. Sin embargo, algunos autores no le 


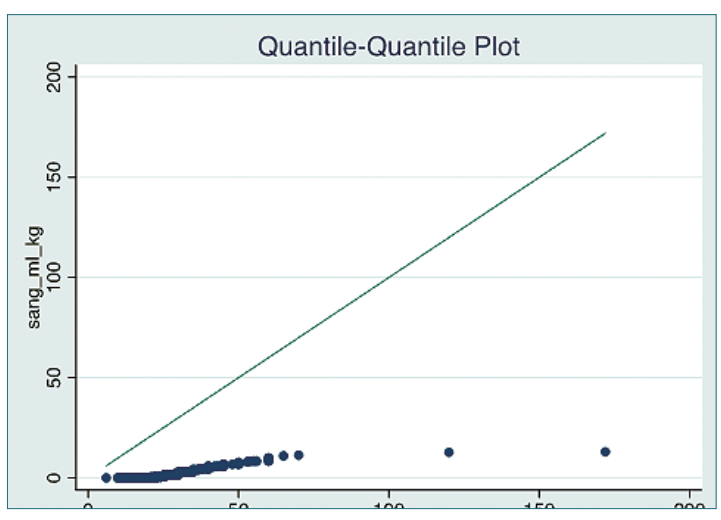

Figura 3. Gráfico Q-Q (cuantil-cuantil) sangrado intraoperatorio en relación a tiempo quirúrgico. A medida que aumenta el tiempo operatorio se observa un ascenso en el sangrado.

confieren mayor aporte a los métodos gravimétricos [19],[20].

Se han descrito múltiples factores asociados al sangrado intraoperatorio en la literatura. En nuestro estudio observamos sólo tres factores asociados de forma significativa con una mayor cuantía del sangrado: La técnica quirúrgica, el uso de propofol y un tiempo quirúrgico más prolongado.

Existe una gran variedad de técnicas quirúrgicas descritas para la amigdalectomía, las cuales son seguras y efectivas. A pesar de esto, la disección fría continúa siendo el procedimiento de elección en la mayoría de los centros. En el último tiempo, la disección caliente ha ganado popularidad, debido a que se asociaría a un menor sangrado intraoperatorio, aunque a expensas de un mayor dolor postoperatorio[15].

Dentro del período evaluado, sólo se realizó un $4 \%$ de amigdalectomías por técnica caliente. Sin embargo, se observó diferencias significativas en relación al sangrado al compararla con la técnica fría, lo que se correlaciona con los hallazgos descritos por otros autores[21]. En un estudio realizado por Aydin et al., en el que se sometió a 40 niños a amigdalectomía a técnica fría y caliente (una a cada lado), se observó que la técnica caliente presentaba un menor sangrado intraoperatorio, mientras que la técnica fría presentó una mejor cicatrización y menor dolor postoperato-

\begin{tabular}{lccc}
\multicolumn{4}{c}{ Tabla 2. Análisis univariado } \\
\hline \multicolumn{1}{c}{ Variable } & Coeficiente & Intervalo de confianza 95\% & p-valor \\
Edad & 0,99 & $0,96-1,03$ & 0,8 \\
Hombre vs mujer & 1,1 & $0,9-1,3$ & 0,3 \\
ASA II vs I & 1,04 & $0,8-1,3$ & 0,7 \\
INR & 1,02 & $0,3-3$ & 0,9 \\
TTPK & 0,99 & $0,97-1,01$ & 0,4 \\
Conteo plaquetario & 1,0 & $0,99-1$ & 0,5 \\
Técnica caliente vs fría & 0,5 & $0,3-0,7$ & 0,001 \\
Amigd+adeno vs aislada & 1,2 & $0,95-1,5$ & 0,1 \\
Inducción Ev. vs Inhalatoria & 0,9 & $0,5-1,6$ & 0,7 \\
Desfluorana vs sevofluorano & 0,9 & $0,1-8$ & 0,9 \\
Fentanilo & 0,96 & $0,89-1,04$ & 0,4 \\
Propofol & 1,3 & $1,1-1,6$ & 0,002 \\
Bloqueador neuromuscular & 0,86 & $0,6-1,3$ & 0,4 \\
Ketoprofeno & 1,1 & $0,8-1,5$ & 0,5 \\
Dexametasona & 2,6 & $0,1-62$ & 0,6 \\
Ácido tranexámico & 1,2 & $0,87-1,6$ & 0,3 \\
Antibióticos & 1,06 & $0,7-1,5$ & 0,75 \\
Intubación nasal vs oral & 1,1 & $0,95-1,3$ & 0,18 \\
Duración de la cirugía & 1,01 & $1,005-1,02$ & 0,002 \\
\hline
\end{tabular}

Modelo de regresión lineal generalizado. 


\begin{tabular}{llll}
\multicolumn{3}{c}{ Tabla 3. Análisis multivariado } \\
\hline \multicolumn{1}{c}{ Variable } & \multicolumn{1}{c}{ Coeficiente } & Intervalo de confianza 95\% & \multicolumn{1}{c}{ p-valor } \\
Técnica caliente vs fría & 0,5 & $0,3-0,7$ & 0,001 \\
Amigd+Adeno vs aislada & 1,5 & $0,7-3,3$ & 0,3 \\
Propofol & 1,3 & $1,1-1,6$ & 0,004 \\
Intubación nasal vs oral & 1,1 & $0,9-1,3$ & 0,4 \\
Duración de la cirugía & 1,02 & $1,01-1,03$ & $<0,00001$ \\
\hline
\end{tabular}

Modelo de regresión lineal generalizado.

rio[22]. En una revisión Cochrane llevada a cabo en 2011, se observó un menor sangrado intraoperatorio con la técnica caliente, pero no se logró establecer superioridad[23]. Algunas técnicas (ej: Harmónico) han surgido en el último tiempo, con el fin de reducir el daño de tejido y producir menor dolor operatorio, sin embargo, continúan siendo utilizadas de forma restringida[24].

El uso de propofol se asoció a un aumento del sangrado intraoperatorio. Algunos estudios in vitro han propuesto un rol antiagregante similar al de la Aspirina[25], lo que podría explicar nuestros resultados. En 1997, De la Cruz et al., publicaron un estudio en donde se observó un efecto antiagregante al administrar propofol a una muestra de sangre[26]. Desde entonces, múltiples estudios surgieron evaluando los efectos del propofol en el sangrado intraoperatorio mostrando resultados contradictorios. Esto se explicaría dado que el propofol tendría un efecto dual: procoagulante mediante la estimulación del efecto de las prostaglandinas y anticoagulante por inhibición de la ciclooxigenasa[27]. En 2008, Okuyucu et al., evaluó el sangrado intraoperatorio según el tipo de mantención de la anestesia general (TIVA propofol vs inhalatoria) determinando un menor sangrado con el uso de propofol[28]. A pesar de los resultados contradictorios, estas diferencias en la técnica anestésica suelen carecer de significado clínico.

El tiempo operatorio se relacionó directamente con un mayor sangrado intraoperatorio, reflejando la ocurrencia de procedimientos más cruentos, o la presencia de procesos amigdalinos crónicos y fibróticos que dificulten la cirugía, como ha sido previamente sugerido por otros autores[9].

En relación a los AINEs, se ha descrito un efecto teórico en la función plaqueteria, que favorecería el sangrado intraoperatorio. Sin embargo, en el presen- te estudio no se observó un mayor sangrado en relación al uso de AINEs. Existen 2 revisiones sistemáticas en las que no se encontró diferencias significativas en la tasa de sangrado al comparar el uso de AINEs vs paracetamol, placebo u opioides[29],[30]. El uso de ketorolaco sería seguro en población pediátrica, pero está asociado a 5 veces más sangrado en población adulta[31].

Se ha descrito que el subgalato reduciría el sangrado y el tiempo operatorio[32]. Sin embargo, fue desestimado durante el análisis debido a que se utilizó de forma indiferenciada de la técnica empleada y de acuerdo a la decisión de cada equipo quirúrgico; el uso de ácido tranexámico también reduciría el sangrado intraoperatorio[33]. Sin embargo, no se encontró un rol protector, posiblemente debido a que su uso fue restringido a aquellos pacientes que presentaron un sangrado de mayor cuantía durante la cirugía.

Las pruebas de coagulación no tuvieron influencia en el sangrado intraoperatorio, y aún en aquellos pacientes con valores moderadamente alterados no presentaron efectos significativos. No hubo necesidad de transfusiones, lo que se correlaciona con lo observado por otros grupos, debido a que en la totalidad de los casos se logra resolver mediante medidas conservadoras[34].

\section{Conclusión}

El sangrado intraoperatorio en la amigdalectomía fue menor a lo descrito en la literatura. De los factores asociados al sangrado, el uso de propofol y un tiempo quirúrgico prolongado fueron factores de riesgo. Mientras que el uso del electrobisturí constituyó un factor protector. 


\section{Referencias}

1. Becke K. Anesthesia for ORL surgery in children. GMS Curr Top Otorhinolaryngol Head Neck Surg. 2014 Dec;13:Doc04. PMID:25587364

2. Stuck BA, Götte $K$, Windfuhr JP, Genzwürker $\mathrm{H}$, Schroten $\mathrm{H}$, Tenenbaum T. Tonsillectomy in children. Dtsch Arztebl Int. 2008 Dec;105(49):852-60. PMID:19561812

3. Rivas Lacarte M. [Tonsillectomy as a major outpatient procedure. Prospective 8-year study: indications and complications. Comparison with inpatients] [in Spanish]. Acta Otorrinolaringol Esp. 2000 Apr;51(3):221-7. PMID:10867396

4. Tan AK, Rothstein J, Tewfik TL. Ambulatory tonsillectomy and adenoidectomy: complications and associated factors. J Otolaryngol. 1993 Dec;22(6):442-6. PMID:8158742

5. Windfuhr J, Seehafer $M$. Classification of haemorrhage following tonsillectomy. J Laryngol Otol. 2001 Jun;115(6):457-61. https://doi. rg/10.1258/0022215011908162 PMID:11429068

6. Myssiorek D, Alvi A. Posttonsillectomy hemorrhage: an assessment of risk factors. Int J Pediatr Otorhinolaryngol. 1996 Sep;37(1):35-43. https://doi.org/10.1016/01655876(96)01364-X PMID:8884405

7. Holden HB, Maher JJ. Some aspects of blood loss and fluid balance in paediatric adenotonsillectomy. BMJ. 1965 Dec;2(5474):134951. https://doi.org/10.1136/ bmj.2.5474.1349 PMID:5848664

8. Manikandan D, Musarrat F, Preetham AP, Anjali R. P. Preetham A, Anjali R. Measurement of Blood Loss during
Adenotonsillectomy in Children and Factors Affecting It. Case Rep Clin Med. 2015;04(05):1516. https://doi.org/10.4236/ crcm.2015.45032.

9. Prasad KC, Prasad SC. Assessment of Operative Blood Loss and the Factors Affecting it in Tonsillectomy and Adenotonsillectomy. Indian J Otolaryngol Head Neck Surg. 2011 Oct;63(4):343-8. https://doi. org/10.1007/s12070-011-02689 PMID:23024939

10. Montague ML, Lee MS, Hussain SS. 'Group and save' for paediatric tonsillectomy: is it required routinely? J Laryngol Otol. 2007 Jun;121(6):55962. https://doi.org/10.1017/ S0022215106005160 PMID:17156577

11. Onotai L, Lilly-Tariah O. Adenoid and tonsil surgeries in children: how relevant is pre-operative blood grouping and cross-matching? Afr J Paediatr Surg. 2013 Jul-Sep;10(3):231-4. https://doi. org/10.4103/0189-6725.120887 PMID:24192465

12. Mann DG, St George C, Scheiner E, Granoff D, Imber P, Mlynarczyk FA. Tonsillectomysome like it hot. Laryngoscope. 1984 May;94(5 Pt 1):677-9. PMID:6371412

13. St Charles CS, Matt BH, Hamilton MM, Katz BP. A comparison of ibuprofen versus acetaminophen with codeine in the young tonsillectomy patient. Otolaryngol Head Neck Surg. 1997 Jul;117(1):7682. https://doi.org/10.1016/ S0194-5998(97)70211-0 PMID:9230328

14. Gabriel P, Mazoit X, Ecoffey C. Relationship between clinical history, coagulation tests, and perioperative bleeding during tonsillectomies in pediatrics. J Clin Anesth. 2000 Jun;12(4):28891. https://doi.org/10.1016/
S0952-8180(00)00164-1

PMID:10960200

15. Robb PJ, Thorning G. Perioperative tranexamic acid in day-case paediatric tonsillectomy. Ann R Coll Surg Engl. 2014 Mar;96(2):127-9. https://doi.org/10.1308/003 $588414 \times 13814021676477$ PMID:24780670

16. Soliman R, Alshehri A. Assessment of the effect of tranexamic acid on perioperative bleeding in pediatric patients undergoing tonsillectomy. Egypt J Anaesth. 2015;31(4):297301. https://doi.org/10.1016/j. egja.2015.07.002.

17. Callanan V, Curran AJ, Smyth DA, Gormley PK. The influence of bismuth subgallate and adrenaline paste upon operating time and operative blood loss in tonsillectomy. J Laryngol Otol. 1995 Mar;109(3):2068. https://doi.org/10.1017/ S0022215100129706 PMID:7745335

18. Knofczynski GT, Mundfrom D. Sample Sizes When Using Multiple Linear Regression for Prediction. Educ Psychol Meas. 2008;68(3):431-42. https:// doi.0.1177/0013164407310131.

19. Eipe N, Ponniah M. Perioperative blood loss assessment - How accurate? Indian J Anaesth. 2006;50(1):35.

20. Johar RS, Smith RP. Assessing gravimetric estimation of intraoperative blood loss. J Gynecol Surg. 1993;9(3):151-4. https:// doi.org/10.1089/gyn.1993.9.151 PMID:10171989

21. Tan GX, Tunkel DE. Control of pain after tonsillectomy in children: a review. JAMA Otolaryngol Head Neck Surg. 2017 Sep;143(9):937-42. https://doi. org/10.1001/jamaoto.2017.0845 PMID:28662233

22. Aydin $\mathrm{S}$, Taskin U, Altas B, Erdil $M$, Senturk T, Celebi $S$, et al. 
Post-tonsillectomy morbidities: randomised, prospective controlled clinical trial of cold dissection versus thermal welding tonsillectomy. J Laryngol Otol. 2014 Feb;128(2):163-

5. https://doi.org/10.1017/ S0022215113003253 PMID:24495415

23. Pinder DK, Wilson $\mathrm{H}$, Hilton MP. Dissection versus diathermy for tonsillectomy. Cochrane Database Syst Rev. 2011 Mar;(3):CD002211. PMID:21412878

24. Pynnonen M BM. Coblation versus other surgical techniques for tonsillectomy (Review). Cochrane Database Syst Rev 2017;(8).

25. Chung HG, Myung SA, Son HS, Kim YH, Namgung J, Cho $M L$, et al. In vitro effect of clinical propofol concentrations on platelet aggregation. Artif Organs. 2013 Jan;37(1):E51-5. https://doi.org/10.1111/ j.1525-1594.2012.01553.x PMID:23145919

26. De La Cruz JP, Carmona JA, Paez MV, Blanco E, Sanchez De La Cuesta F. Propofol inhibits in vitro platelet aggregation in human whole blood. Anesth Analg. 1997 Apr;84(4):919-21. https:// doi.org/10.1213/00000539199704000-00040 PMID:9085982
27. Hirakata $H$, Nakamura $K$, Yokubol B, Toda $\mathrm{H}$, Hatano $\mathrm{Y}$, Urabe $\mathrm{N}$, et al. Propofol has both enhancing and suppressing effects on human platelet aggregation in vitro. Anesthesiology. 1999 Nov;91(5):1361-9. https:// doi.org/10.1097/00000542199911000-00028 PMID:10551587

28. Okuyucu S, Inanoglu K, Akkurt CO, Akoglu E, Dagli S. The effect of anesthetic agents on perioperative bleeding during tonsillectomy: propofol-based versus desflurane-based anesthesia. Otolaryngol Head Neck Surg. 2008 Feb;138(2):15861. https://doi.org/10.1016/j. otohns.2007.10.032 PMID:18241708

29. Riggin L, Ramakrishna J, Sommer DD, Koren G. A 2013 updated systematic review \& meta-analysis of 36 randomized controlled trials; no apparent effects of non steroidal antiinflammatory agents on the risk of bleeding after tonsillectomy. Clin Otolaryngol. 2013 Apr;38(2):115-29. https:// doi.org/10.1111/coa.12106 PMID:23448586

30. Lewis SR, Nicholson A, Cardwell ME, Siviter G, Smith AF. Nonsteroidal anti-inflammatory drugs and perioperative blee- ding in paediatric tonsillectomy. Cochrane Database Syst Rev. 2013 Jul;(7):CD003591. https:// doi.org/10.1002/14651858. CD003591.pub3 PMID:23881651

31. Chan DK, Parikh SR. Perioperative ketorolac increases post-tonsillectomy hemorrhage in adults but not children. Laryngoscope. 2014 Aug;124(8):1789-93. https://doi.org/10.1002/ lary.24555 PMID:24338331

32. Fenton JE, Blayney AW, O'Dwyer TP. Bismuth subgallate-its role in tonsillectomy. J Laryngol Otol. 1995 Mar;109(3):2035. https://doi.org/10.1017/ S002221510012969X PMID:7745334

33. Robb PJ, Thorning G. Perioperative tranexamic acid in day-case paediatric tonsillectomy. Ann R Coll Surg Engl. 2014 Mar;96(2):127-9. https://doi.org/10.1308/003 588414X13814021676477 PMID:24780670

34. Adekola OO, Akanmu ON, Bamigboye BA, Akinnola MD, Adedolapo HL, Dada OI, et al. How frequent is homologous blood transfusion during pediatric adenoidectomy and tonsillectomy? J Clin Sci. 2016;13(4):187-92. https://doi.org/10.4103/24686859.192298. 